
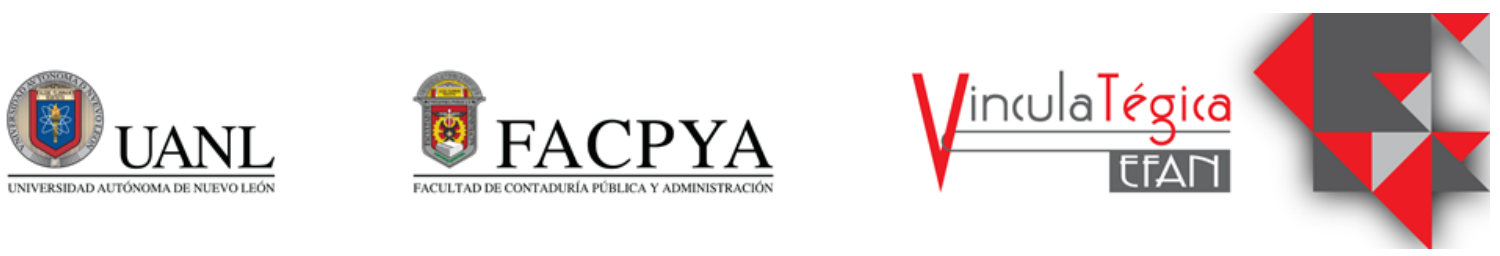

\title{
Factores que influyen en la adquisición de un departamento en Monterrey (Nuevo León)
}

\author{
Brenda Carolina Bueno Torres ${ }^{1}$ y Evelyn Magdalena Rodríguez Presa ${ }^{2}$ \\ ${ }^{1}$ Estudiante de Posgrado de la Maestría en Valuación de la Facultad de Arquitectura en la Universidad \\ Autónoma de Nuevo León, brendacbt.16@gmail.com, Av. Universidads/n, Ciudad Universitaria, San Nicolás \\ de los Garza, N.L., C.P.66455. \\ ${ }^{2}$ Profesor e investigador de la Facultad de Arquitectura en la Universidad Autónoma de Nuevo León, \\ evelynpresa@gmail.com, Av. Universidad s/n, Ciudad Universitaria, San Nicolás de los Garza, N.L., \\ C.P.66455.
}

Información del artículo revisado por pares

Fecha de aceptación: junio-2021

Fecha de publicación en línea: diciembre-2021

DOI: https://doi.org/10.29105/vtga7.1-83

\begin{abstract}
Resumen
Este artículo presenta información y datos que son importantes de considerar al momento de adquirir un bien inmueble, específicamente un departamento en el municipio de Monterrey, Nuevo León. El objetivo central de la investigación es analizar los principales factores que se toman en cuenta antes de adquirir un departamento, revisando las variables obtenidas a través de una encuesta realizada y aplicada a 122 personas vía Google forms. La finalidad de este artículo será evaluar los principales factores que se toman de cuenta antes de realizar una inversión, utilizando un muestreo no probabilístico por conveniencia y el análisis de las variables que se obtuvieron. La hipótesis que se intenta probar es que, para poder adquirir un departamento, se tienen que validar y revisar ciertos puntos importantes y ver cómo se comportan esos mismos factores en la toma de decisión de las personas.
\end{abstract}

Palabras clave: análisis, departamento, inversión inmobiliaria, mercado inmobiliario, Monterrey.

\begin{abstract}
This article presents information and data that are important to consider when purchasing real estate, specifically an apartment in the municipality of Monterrey, Nuevo León. The main objective of the research is to analyze the main factors that are considered before acquiring an apartment, reviewing the variables obtained through a survey carried out and applied to 122 people via Google forms. The purpose of this article will be to evaluate the main factors that are considered before making an investment, using a non-probability sampling for convenience and the analysis of the variables that were obtained.

The hypothesis being tested is that, to acquire an apartment, certain important points have to be validated and reviewed and see how those same factors behave in people's decision-making.
\end{abstract}

Keywords: analysis, department, real estate investment, real estate market, Monterrey.

JEL: H43, L85, L74. 


\section{INTRODUCCIÓN}

Invertir en el mercado inmobiliario, sobre todo en la adquisición de una propiedad propia es sin lugar a duda una de las decisiones más importantes que puede tomar cualquier persona o familia, ya que brinda seguridad para el patrimonio de las personas.

Con el paso del tiempo la ciudad de Monterrey se ha ido llenando de proyectos verticales de usos mixtos que cuentan con área habitacional, comercial y en algunos casos oficinas. Una gran cantidad de proyectos de despachos y de arquitectos reconocidos se están volviendo iconos de la ciudad, fomentando cada vez más la decisión de comprar un departamento en lugar de una casa habitación, por lo que hoy en día existen muchas opciones y una gran competencia de diversas desarrolladoras inmobiliarias para que las personas puedan elegir el tipo de departamento más conveniente de acuerdo con sus necesidades.

Recientemente, la vivienda vertical se ha convertido en una opción muy común para vivir en el municipio de Monterrey, Nuevo León, lugar que cuenta con una gran variedad de proyectos verticales de usos mixtos para poder invertir, desde desarrollos nivel mediobajo hasta nivel alto o proyectos integrales que se vuelven pequeñas ciudades en un mismo complejo, contando con proyectos construidos con alta tecnología, con una gran cantidad de características, que se pueden adaptar a los diferentes gustos y preferencias de los compradores.

Por lo tanto, Monterrey es una de las ciudades con mayor desarrollo y crecimiento inmobiliario, convirtiéndose en la ciudad más moderna en el norte del país específicamente en este tipo de edificios de usos mixtos, que brindan al comprador comodidad y satisfacción de todas sus necesidades en un mismo lugar. (Vázquez, 2018).

Nuestro municipio está pasando por la misma situación que las otras grandes ciudades del país, debido a la dinámica demográfica de la propia urbe y su crecimiento constante, por lo que el encarecimiento de la tierra se vuelve mucho más común, principalmente de los terrenos ubicados en las zonas que continuamente cambian su uso de suelo a multifamiliar, comercial y de servicios.

Estos cambios ocasionan el incremento de la demanda de departamentos, debido a que es una buena opción para vivir en las zonas de mayor plusvalía. (Vázquez, 2018).

Entonces la creciente demanda por una vivienda y la ineficacia de la planeación en la ciudad ha sido necesario buscar y establecer nuevas formas para construir, dados los aspectos sociales, urbanos, geográficos, ecológicos que permitan el desarrollo de las familias en un entorno adecuado y agradable. (Moreno y Alvarado, 2011).

En este sentido, adquirir un departamento tiene múltiples beneficios identificados por la población, por lo que se ha convertido en unos de los principales tipos de inversión inmobiliaria, algunas de las virtudes de los departamentos es que es posible vivir en un mismo edificio debido a que cuentan, generalmente con áreas comunes como jardines y áreas verdes, zonas de esparcimiento y particularidades que cada desarrolladora pueda ofrecer de acuerdo con la zona en la que se ubique el complejo.

Es importante mencionar que, si el proyecto en donde se desea invertir tiene más características deseadas comúnmente por los compradores, tendrán precios más altos y por ende si cuenta con menos cualidades tendrán precios más bajos, agregando también un factor muy importante que es la ubicación que tenga dicho proyecto. (Daúl, 2019).

Antes de realizar una inversión de esta magnitud, es importante validar distintas opciones y considerarlas para después tomar la decisión. Inicialmente es necesario garantizar la cobertura de las necesidades, asegurarse que dicha propiedad cumple con sus expectativas de correcta y adecuada inversión patrimonial a realizar.

El trabajo se organiza como sigue. En la sección dos se presenta una breve revisión teórica del tema. En la sección tres se describe el método para la obtención de los datos del presente artículo. En la sección cuatro, se muestran los resultados obtenidos, así como su análisis y explicación, por último, se presentan algunas conclusiones y referencias. 


\section{MARCO TEÓRICO}

\subsection{Edificación vertical}

Hoy en día existe un impulso hacia la vivienda vertical por parte del gobierno y de las desarrolladoras inmobiliarias en las grandes ciudades como Monterrey, Guadalajara o Ciudad de México, por lo que cada vez hay una mayor demanda por este tipo de vivienda. (Valdés, 2017).

La construcción de vivienda vertical específicamente en Monterrey y su área metropolitana está en su punto máximo, puesto que, en la actualidad, alrededor del $25 \%$ de las personas que busca un hogar en los segmentos medio y alto, prefiere adquirir un departamento. (Chapa,2018).

El mercado de la vivienda se define como un segmento importante de la economía urbana. Las preferencias de los consumidores se pueden entender básicamente por los precios de las viviendas que desean adquirir.

Las propiedades que ofrecen un mayor número de cualidades y virtudes deseadas por los clientes tendrán precios más altos, por ende, las que tengan menos características tendrán precios mucho más bajos. (Poeta, 2019).

La edificación vertical ha marcado la pauta en la construcción urbana y se ha convertido en tendencia en espacios para vivir, trabajar y convivir socialmente en el municipio de Monterrey, Nuevo León (Juárez, 2017).

La problemática de la urbanización es un asunto de carácter federal, ya que, de acuerdo con informes del INEGI desde el año 2016, a nivel nacional actualmente ocho de cada diez habitantes viven en localidades urbanas de más de 2,500 habitantes y crecen en forma desproporcionada y sin planificación alguna (García, 2017).

La redensificación de la ciudad es muy importante en la medida en que se mezclen ciertas actividades, personas o usuarios y tiempos; de esta forma se potencializa de una mejor manera la construcción de escenarios alternativos de uso, frecuencia y aprovechamiento de la infraestructura territorial, para lograr un mejor desarrollo y diseño (Gómez, 2014).
Sin duda cada ciudad es única y posee sus propios factores que ejercen una influencia decisiva e importante en su planeamiento, por lo que las autoridades de la ciudad deben involucrarse de manera distinta según los diferentes niveles en el proceso de toma de decisiones. (Machado, 2004).

El proceso de crecimiento y de planeación urbana se han realizado en la mayoría de las ocasiones, sin límites claros de diseño y sin seguir una pauta, lo cual, con el paso del tiempo, ha ocasionado que los desarrollos inmobiliarios no cuenten con la adecuada provisión de servicios educativos, de salud, infraestructura, conectividad y cercanía a las fuentes de empleo, que brinden calidad de vida a la población y que no genere largos desplazamientos (Gonzáles, 2017).

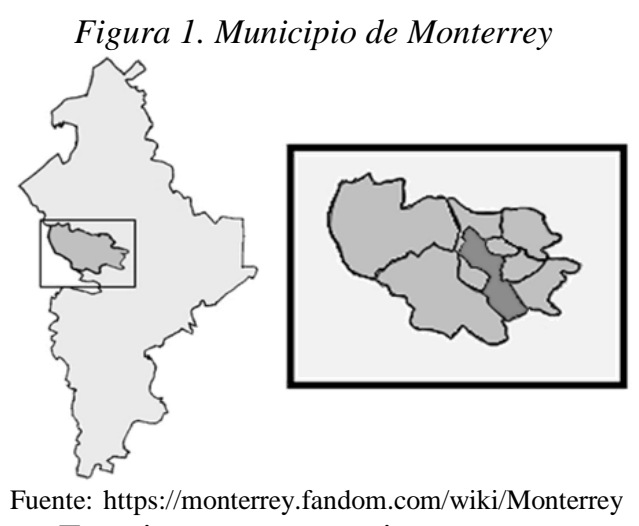

Es importante reiterar que este crecimiento acelerado en la construcción de edificios se ha debido también a la dinámica demográfica y a la falta de espacios dentro de la ciudad para poder crear desarrollos de vivienda, (Juárez, 2017). En este contexto, se suman factores como el problema de la movilidad y la falta de espacios públicos, en las periferias. Ahora bien, el diseño de nuevas formas de desarrollos de proyectos es necesario para crear nuevos espacios para vivir y trabajar, revisando de qué manera puede crecer la ciudad de una mejor manera, por lo que los dueños de las grandes desarrolladoras inmobiliarias han apostado por esta manera de construir ofreciendo espacios que se adapten a las necesidades de cualquier cliente $o$ consumidor.

El proceso de verticalización de una ciudad modifica sin lugar a duda el espacio urbano porque redefine el valor y el uso del 
suelo y al mismo tiempo altera las relaciones sociales entre los hombres y el medio ambiente urbanizado. La relación social, está íntimamente ligada a la idea del ascenso social, de seguridad, de confort y modernidad (Machado, 2004).

Para que el diseño de la vivienda vertical incida positivamente en la calidad de vida de las personas, debe abordarse desde tres ángulos principales: ciudad, espacio y usuario (Gómez, 2014).

Otro factor que influye en crear edificios verticales es la mala calidad del aire $\mathrm{y}$ ambiental en las grandes urbes, por lo que los especialistas como urbanistas, arquitectos e ingenieros dan su visto bueno para la construcción de ciudades verticales como modelos de desarrollo sostenible (Bautista, 2017).

En este contexto, la construcción de nuevos desarrollos verticales dentro de las ciudades ayuda en gran medida a reducir las emisiones de dióxido de carbono, lograr la eficiencia energética, aprovechar el agua pluvial y a contar con un adecuado manejo de los desechos que se generen por lo que se prevé que una ciudad vertical podría ahorrar hasta un $75 \%$ de energía y reducir hasta en un 90\% las emisiones contaminantes de las ciudades (Valdés, 2017).

Las desarrolladoras inmobiliarias siguen creando viviendas verticales y ésta continúa reinventándose, buscando elevar el nivel de sus habitantes al mismo tiempo que es una muy buena solución para el desarrollo urbano al ofrecer más viviendas y áreas habitacionales, rentables en términos de espacio, así como la disminución del costo de todos los servicios públicos para incrementar el bienestar y la calidad de vida de los clientes. (Juárez, 2017).

$\mathrm{Si}$ se comienza a ejercer un planeamiento urbano bien elaborado, es decir el desarrollo esperado por las autoridades, se elevará el nivel de calidad de vida de los ciudadanos, con un mejoramiento en las áreas de infraestructura, espacios públicos, áreas verdes, medio ambiente urbano, destino de los residuos, uso de suelo, violencia, seguridad, entre otros (Machado, 2004).
Los rascacielos y edificios verticales sin duda se han convertido en la mejor alternativa para atender los requerimientos de la relación que existe entre la construcción y el medio ambiente, debido a que se implementan nuevas tecnologías que ayudan al ahorro de energía y equilibrio con su entorno, y se concluye que en la actualidad son la solución a problemas como la sobrepoblación. (Juárez, 2017).

\subsection{Ventajas de vivir en un desarrollo vertical}

Como se mencionaba anteriormente, adquirir un departamento hoy en día se ha convertido en el tipo de vivienda ideal para muchas personas cuyo principal objetivo es vivir en un lugar que brinde comodidad, que cuente con excelentes espacios y sobre todo una buena ubicación.

El lado positivo de las viviendas verticales es que se puede potenciar al máximo el uso del suelo mediante el aprovechamiento de los espacios, el consumo eficiente de los servicios como agua, luz, basura, teléfono y drenaje, además del transporte público. (González, 2017).

Existen muchas otras ventajas que ofrece el vivir en un departamento dentro de un desarrollo vertical de usos mixtos en comparación con las de una casa habitación normal en un fraccionamiento privado o en una colonia, entre las cuales podemos mencionar las siguientes:

i. Mejor aprovechamiento de suelo

ii. Suprimir largos recorridos

iii. Desarrollo vecinal más fuerte

iv. Preservar espacios abiertos

v. Buena ubicación

vi. Cercanía de servicios

vii. Fomentan la convivencia de vivirtrabajar en un mismo espacio

viii. Fomentan la convivencia sin el uso del automóvil (Valdés, 2017).

Construir de manera vertical es una de las soluciones para poder lograr con el paso del tiempo un crecimiento urbano ordenado y en equilibrio con el medio y es la solución más viable para poder atender el problema del crecimiento acelerado de la población. (Bautista, 2017). 


\subsection{Adquisición de un bien inmueble}

El momento de realizar una compra no puede llevarse a cabo solamente tomando en cuenta los factores individuales como, por ejemplo: el precio, la ubicación, el diseño del inmueble, así como también la opinión de los familiares, la tranquilidad, la seguridad, accesibilidad, entre otras. (Daúl, 2019).

De acuerdo con lo anterior, los principales factores a considerar en la compra de un bien inmueble, en el caso de un departamento, son el precio y la calidad del bien, así como la disponibilidad de los servicios básicos.

El precio se determina como uno de los principales factores para la toma de decisiones ya que el presupuesto de los consumidores es decisivo para la adquisición de un producto (Santos y Lima, 2012).

Existen otras características que también son importantes a considerar al momento de adquirir un departamento como por ejemplo las características de la zona en la estará ubicado el departamento, tomar en cuenta los alrededores, las vialidades, accesos, así como el equipamiento como escuelas hospitales y centros comerciales.

Además, es necesario considerar las necesidades de las personas en cuanto al espacio, es decir, el número de integrantes de la familia $\mathrm{y}$, por lo tanto, la cantidad de habitaciones y áreas con los que cuente dicho inmueble, etcétera.

Por último, se requiere evaluar también la capacidad económica para poder adquirir dicho departamento y ver cuál será el tipo de financiamiento que se utilizará y que más se apegue a las necesidades y condiciones del comprador.

Para concluir, el formato de la vivienda vertical ha tenido un boom en el municipio de Monterrey en los últimos 10 años, por lo que se espera que esta actividad siga en aumento por el cambio de estilo de vida de las generaciones jóvenes, quienes prefieren adquirir o comprar un departamento que ya cuente con seguridad, amenidades y tener el mantenimiento resuelto, al mismo tiempo buscan vivir lo más cerca de su trabajo para evitar la pérdida de tiempo en traslados. (Garza, 2018).

\section{MÉTODO}

Para este artículo se utilizó un muestreo no probabilístico por conveniencia, aplicándose una encuesta de manera digital mediante Google Forms a 122 personas (número de personas que respondieron), la cual consta de 22 preguntas y 3 secciones. La primera sección es referente a la información general de los encuestados, la segunda cuenta con preguntas con respecto a ciertas preferencias al adquirir un departamento y la tercera son preguntas acerca de las características que tendría el departamento que les gustaría adquirir.

Con base en las respuestas, se eligieron las variables a analizar, posteriormente dichas variables se vaciaron en una base de datos la cual se integró al programa de Paquete Estadístico para las Ciencias Sociales (Statistical Package for the Social Sciences), utilizado para la realización del artículo.

\section{RESULTADOS}

Con base en la información obtenida de las encuestas aplicadas, se obtuvieron resultados importantes para determinar los principales factores que influyen en la toma de decisión al adquirir un departamento en el municipio de Monterrey, Nuevo León.

De esta manera y utilizando los resultados de instrumento, se presentan las siguientes tablas. A manera de aclaración, en dichas tablas hay algunas variables que tienen un total por debajo a los 122 encuestados, esto debido a omisiones en respuesta.

En la Tabla 1, llamada Frecuencias, específicamente de las preferencias de las personas encuestadas, se reporta el número de veces que se repiten las respuestas en cada pregunta y se puede observar que de las 122 personas a quienes se les hizo llegar la encuesta, el 54\% está interesado en adquirir un departamento en el municipio de Monterrey el día de hoy.

La principal razón por la cual cerca de la mitad de los encuestados no está interesado se debe a que varios encuestados preferían una casa habitación o piensan que vivir en uno de los tantos edificios de usos mixtos que se encuentran en el municipio y consideran que vivir en un departamento puede ser muy 
costoso, tanto el mantenimiento como el precio de este.

Tabla 1. Preferencias de los encuestados

\begin{tabular}{lll}
\hline Interés en comprar & Frecuencia & Porcentaje \\
\hline Sí & 66 & 54.10 \\
No & 56 & 45.90 \\
Total & 122 & 100.00 \\
\hline Finalidad & & \\
\hline Inversión & 60 & 49.20 \\
Vivir & 35 & 28.70 \\
Rentar & 26 & 21.30 \\
Total & 121 & 99.20 \\
\hline Ubicación & & \\
\hline Zona Centro & 50 & 41.00 \\
Zona Sur & 31 & 25.40 \\
Zona Cumbres & 22 & 18.00 \\
Zona Country & 19 & 15.60 \\
Total & 122 & 100.00 \\
\hline Categoría & & \\
\hline Medio & 22 & 18.00 \\
Residencial & 89 & 73.00 \\
Residencial plus & 9 & 7.40 \\
Total & 120 & 98.40 \\
\hline Compañía & & \\
\hline Solo (a) & 50 & 41.00 \\
Con mi pareja & 46 & 37.70 \\
Con mi familia & 26 & 21.30 \\
Total & 122 & 100.00 \\
\hline Financiamiento & & \\
\hline Crédito bancario & 96 & 78.70 \\
INFONAVIT & 17 & 13.90 \\
FOVISSTE & 9 & 7.40 \\
Fuente: Elaboración propia & 122 & 100.00 \\
\hline & & \\
\hline & & \\
\hline & & \\
\hline
\end{tabular}

En caso de adquirir un departamento, el $41 \%$ de los encuestados se inclina por uno ubicado en el centro de la ciudad. Si bien desde hace algunos años los inversionistas han decidido apostar por construir edificios en el centro para reactivar la zona seguido de la Zona Sur con un $25 \%$, Zona Cumbres con un $18 \%$ y la Zona Country con el $16 \%$.

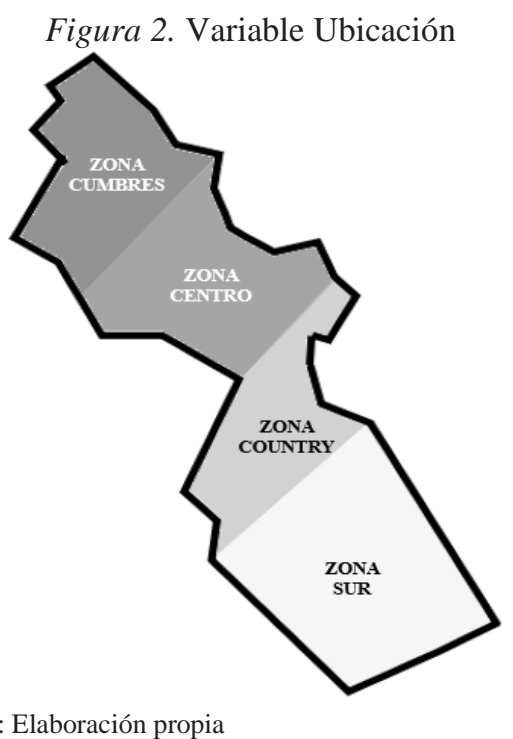

En la Figura 3 se muestra la gráfica de frecuencia de la variable finalidad, se concluye que el $50 \%$ de las personas encuestadas adquirirían un departamento como inversión, seguido del $29 \%$ cuya finalidad es vivir en él y el resto que equivale a un $21 \%$ para rentarlo.

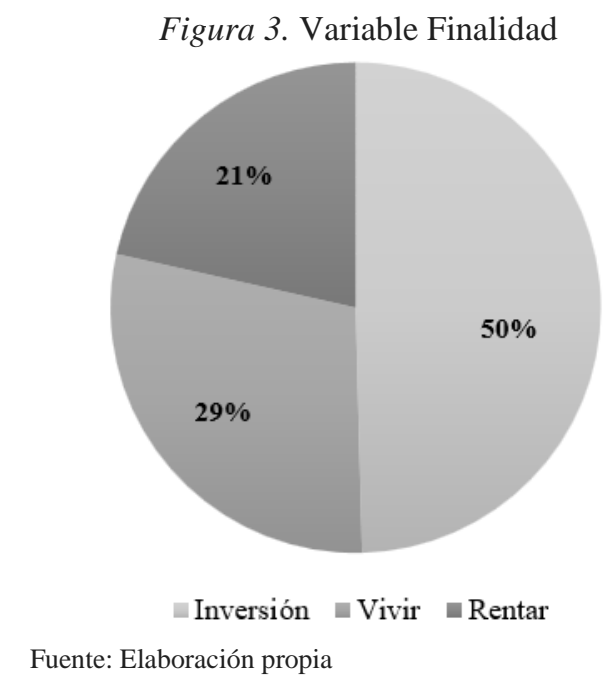

Con respecto al tipo de departamento que les interesa adquirir al cerca de tres cuartas partes de la muestra se interesa porque sea de categoría residencial, seguido la categoría media con $18 \% \mathrm{y}$, por último, el residencial plus con un $7 \%$.

Una variable importante para tomar en cuenta al momento de adquirir una propiedad 
es sin duda el tipo de financiamiento y la forma de pago que se utilizará para poder solventar esa gran inversión.

Una de las preguntas en la encuesta aplicada fue precisamente el tipo de financiamiento que se utilizará, siendo la respuesta más escogida por los encuestados financiarse a través de créditos bancarios.

Aunado a lo anterior, podemos observar en la Figura 4 las preferencias de cada una de las opciones y se concluye que, el $79 \%$ de las personas encuestadas prefieren un crédito bancario, seguido del $14 \%$ que corresponde al INFONAVIT y el 7\% con FOVISSTE.

Como consecuencia de la pandemia que acontece a nivel mundial, las tasas de interés en los bancos han tenido cambios y variaciones en los últimos meses, por lo que los bancos ofrecen diferentes tasas que se pueden adaptar a las necesidades del comprador, concluyendo que este factor puede haber influido en la decisión de considerar el crédito bancario como la principal forma de financiamiento.

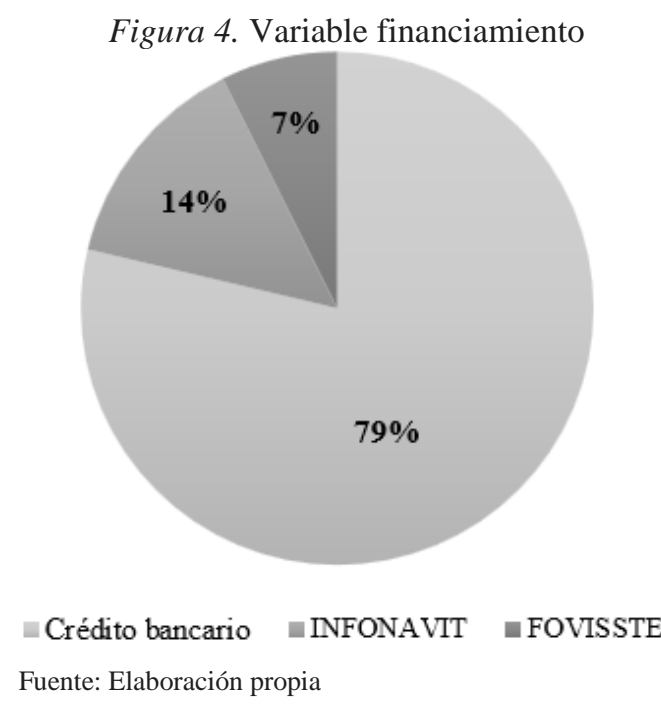

Continuando con el análisis de los datos, en la siguiente tabla se muestran las frecuencias y porcentajes de las variables que hacen referencia a las características del departamento a adquirir. Se puede observar que el $41 \%$ de los encuestados están interesados en adquirir un departamento con un precio que ronda de 1 a 1.5 MDP (Millones de pesos), seguido del precio de 1.5 a 2 MDP (Millones de pesos) con un 36\%.
Otro factor por considerar son los espacios que tendrá el inmueble y ver como se adapta a la cantidad de personas que vivirían en él. En la Tabla 2 se puede observar que el $44 \%$ de los encuestados desean que su departamento cuente con dos recámaras, seguido del $33 \%$ con tres recámaras y el $3 \%$ con más de tres recámaras.

Tabla 2. Características del departamento

\begin{tabular}{lll}
\hline Precio & Frecuencia & Porcentaje \\
\hline Menos de 1 MDP & 17 & 13.90 \\
De 1 a 1.5 MDP & 50 & 41.00 \\
De 1.5 a 2 MDP & 44 & 36.10 \\
De 2 a 3 MDP & 10 & 8.20 \\
Total & 121 & 99.20 \\
\hline Recámaras & & \\
\hline 2 recámaras & 53 & 43.40 \\
3 recámaras & 40 & 32.80 \\
Más de 3 recámaras & 3 & 2.50 \\
Total & 96 & 78.70 \\
\hline Áreas comunes & & \\
\hline Casa club y alberca & 34 & 27.90 \\
Área de juegos & 3 & 2.50 \\
Áreas verdes & 25 & 20.50 \\
exteriores & 17 & 13.90 \\
Asadores y terrazas & 1 & 0.80 \\
Canchas de juegos & 1 & 13.10 \\
Gimnasio & 16 & 78.70 \\
Total & 96 & \\
\hline Equipamiento & & 31.10 \\
\hline Supermercados & 23 & 4.90 \\
Centros comerciales & 38 & 23.80 \\
Escuelas & 6 & 78.70 \\
Parques y áreas & 29 & \\
verdes & 96 & \\
Total & & \\
\hline Fuente: Elaboración propia & & \\
\hline
\end{tabular}

En la Figura 5 se muestra la gráfica de frecuencia de la variable de áreas comunes que más les gustaría tener a las personas encuestadas. Respecto a las áreas comunes, se concluye que el $28 \%$ de las personas 
encuestadas prefieren una casa club y alberca en el completo de usos mixtos, seguido de un $21 \%$ que se refiere a las áreas exteriores, un $14 \%$ con asadores y terrazas y un $13 \%$ se inclina por la opción de un gimnasio.

Figura 5. Áreas comunes

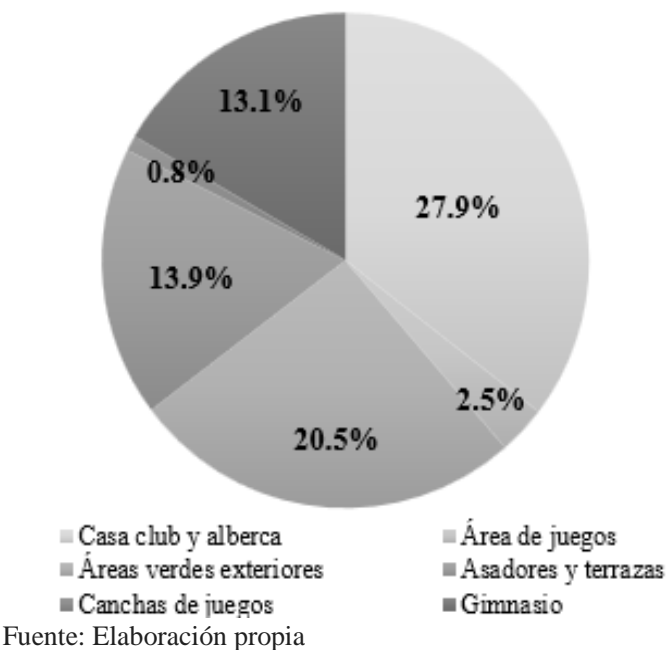

Otra variable importante es la infraestructura que se ubica cerca del lugar donde estará el departamento. Algunas de las opciones que se muestran en la encuesta utilizada fueron los centros comerciales, con $31 \%$ de las preferencias, seguido de parques y áreas verdes con un $24 \%$, supermercados con el $19 \%$ y finalmente las escuelas con un $5 \%$.

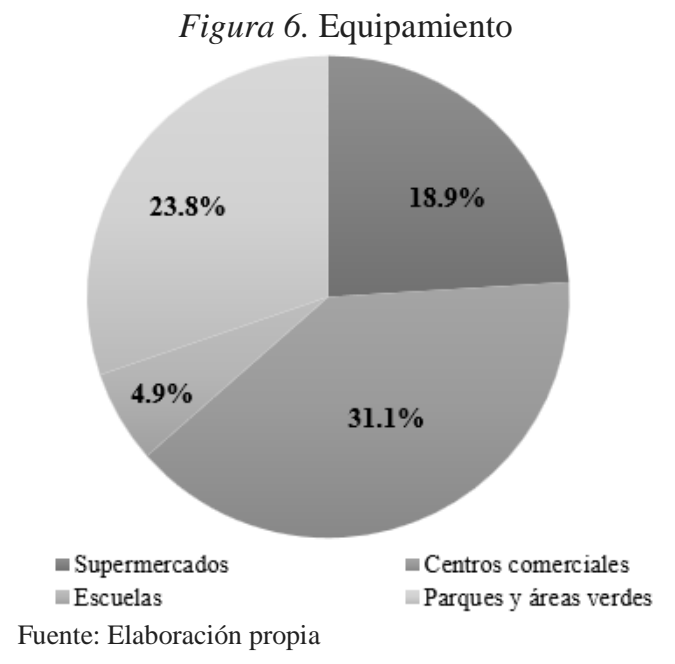

Continuando con el análisis de los datos, se muestrea a continuación la Tabla 3, la cual muestra las variables que se analizaron y se describe cada una de ellas.
Tabla 3. Descripción de variables

\begin{tabular}{ll}
\hline Variables & Descripción \\
\hline Edad & $\begin{array}{l}\text { Cantidad de años de las } \\
\text { personas encuestadas. }\end{array}$ \\
\hline Género & $\begin{array}{l}\text { Identidad sexual de los } \\
\text { encuestados. Variable dummy }\end{array}$ \\
\hline Estado civil & $\begin{array}{l}\text { Situación jurídica de las } \\
\text { personas encuestadas. }\end{array}$ \\
\hline Escolaridad & $\begin{array}{l}\text { Nivel de estudios de los } \\
\text { encuestados. }\end{array}$ \\
\hline Ingreso salarial & $\begin{array}{l}\text { Salario promedio mensual de las } \\
\text { personas encuestadas. }\end{array}$ \\
\hline Residencia & $\begin{array}{l}\text { Lugar del AMM en el que viven } \\
\text { los encuestados. }\end{array}$ \\
\hline Interés en & $\begin{array}{l}\text { Interés en comprar un } \\
\text { departamento. Variable dummy, }\end{array}$ \\
\hline comprar & $\begin{array}{l}\text { Finalidad de adquirir un } \\
\text { departamento. }\end{array}$ \\
\hline Ubicación & $\begin{array}{l}\text { Zona donde se ubica el } \\
\text { departamento a comprar. }\end{array}$ \\
\hline Categoría & $\begin{array}{l}\text { Categoría del departamento a } \\
\text { comprar. }\end{array}$ \\
\hline Compañía & $\begin{array}{l}\text { Personas con las que viviría en } \\
\text { el departamento. }\end{array}$ \\
\hline Financiamiento & $\begin{array}{l}\text { Tipo de financiamiento a } \\
\text { utilizar para adquirir el } \\
\text { departamento. }\end{array}$ \\
\hline Precio & Precio del departamento. \\
\hline Recámaras & $\begin{array}{l}\text { Cantidad de recámaras del } \\
\text { departamento. }\end{array}$ \\
\hline Áreas comunes & $\begin{array}{l}\text { Áreas comunes que preferirían } \\
\text { tener en el edificio de } \\
\text { departamentos. }\end{array}$ \\
\hline $\begin{array}{l}\text { Equipamiento cerca del edificio } \\
\text { que los encuestados consideran } \\
\text { para comprar su departamento. }\end{array}$ \\
\hline propia
\end{tabular}

A continuación, se muestra la Tabla 4, referente a los estadísticos descriptivos de las variables analizadas, se observa que la edad promedio de los encuestados es de 39.67 años.

La variable de interés en comprar un departamento obtuvo una media del 1.46, con un mínimo de 1 y un máximo de 2 .

En cuanto la finalidad principal por la cual se adquiriría un departamento en estos tiempos, el promedio es de 1.72 siendo la medida de mínima de 1 y la máxima de 3.

Así mismo, la variable del tipo financiamiento a utilizar para adquirir el departamento cuenta con un promedio de 1.29. 
Tabla 4. Estadísticos descriptivos de las variables

\begin{tabular}{lllll}
\hline Variables & Media & Desviación & Mín. & Máx. \\
\hline Edad & 39.67 & 13.71 & 21 & 64 \\
$\begin{array}{l}\text { Interés en } \\
\text { comprar }\end{array}$ & 1.46 & 0.50 & 1 & 2 \\
Finalidad & 1.72 & 0.80 & 1 & 3 \\
Ubicación & 2.08 & 1.10 & 1 & 4 \\
Categoría & 1.89 & 0.50 & 1 & 3 \\
Compañía & 1.80 & 0.77 & 1 & 3 \\
Financiamiento & 1.29 & 0.60 & 1 & 3 \\
Precio & 2.39 & 0.83 & 1 & 4 \\
Recámaras & 2.48 & 0.56 & 2 & 4 \\
Áreas comunes & 2.96 & 1.18 & 1 & 6 \\
Equipamiento & 2.43 & 1.16 & 1 & 4 \\
\hline
\end{tabular}

Fuente: Elaboración propia

La variable de ubicación, es decir la zona en la que estará ubicado el departamento a elegir tiene un promedio de 2.08 .

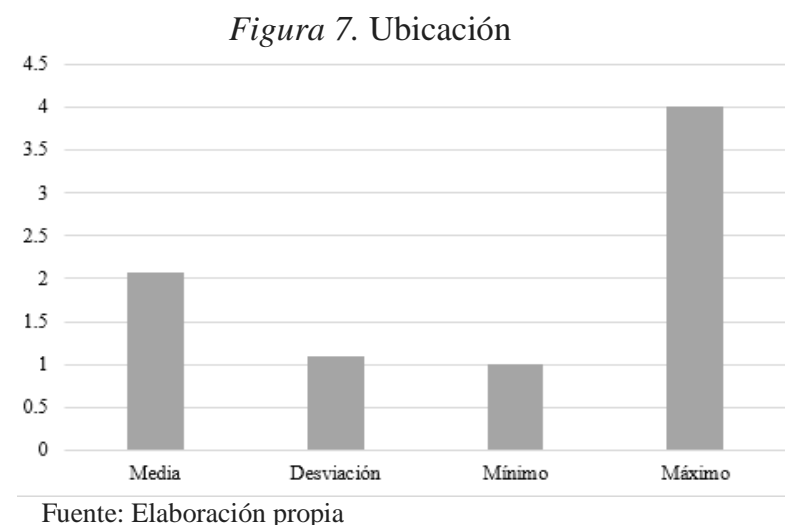

La siguiente variable por analizar es la de categoría del departamento, teniendo un promedio de 1.89 , siendo el mínimo de 1 y un máximo de 3.

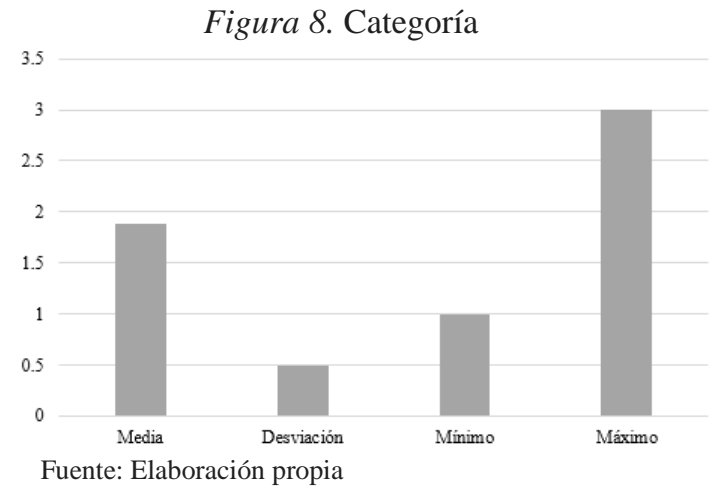

La siguiente variable es el precio del departamento, dónde se obtuvo un promedio de 2.39, siendo el mínimo 1 y un máximo de 4.

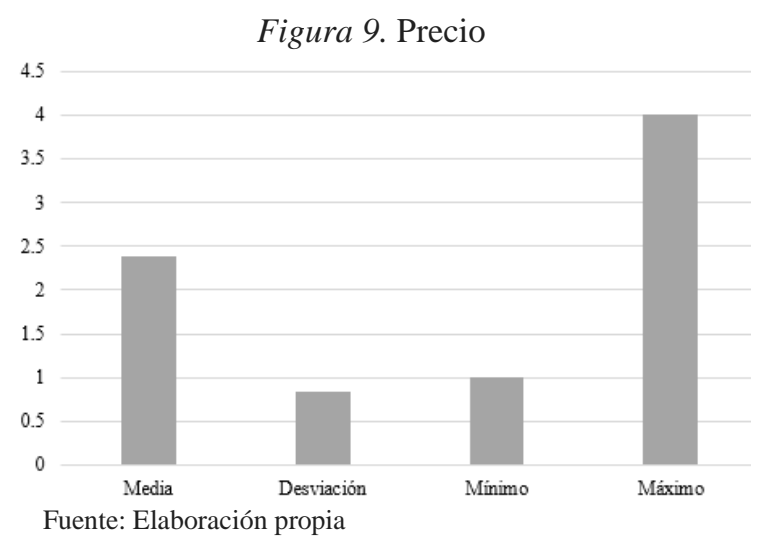

En la siguiente figura se muestra la variable de la cantidad de recámaras deseadas en el departamento, con un promedio de 2.48 , siendo el mínimo 2 y un máximo de 4 .

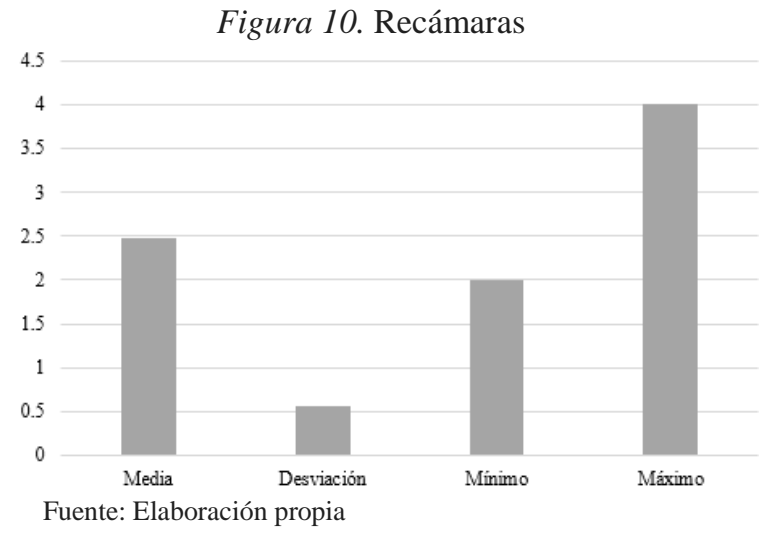

Por último, al analizar los datos de la variable de áreas comunes y equipamiento se observa que el promedio es de 2.96 y 2.43 respectivamente.

\section{CONCLUSIONES}

En las grandes urbes, la adquisición de un departamento se está volviendo más común en lugar de adquirir una vivienda, principalmente por la gran oferta que existe en la ciudad y por la ubicación de los complejos de usos mixtos, los cuales ofrecen a las residentes áreas para vivir de una manera más cómoda.

De acuerdo con los resultados el 54\% de los encuestados están interesados en adquirir un departamento, cabe mencionar que la ubicación que más demanda tiene en base a los 
resultados de las encuestas, es la zona centro del municipio, la cual facilitaría mucho el desplazamiento de las personas, debido a que la ciudad está en constante crecimiento y hoy en día la mayoría de las colonias de vivienda se encuentran a las afueras o en municipios colindantes a Monterrey, por lo que acercarse más al centro es una buena opción que considerar para vivir.

Algunos de los principales factores que juegan un papel importante al momento de adquirir este tipo de vivienda son los siguientes: la ubicación del departamento, el precio de este, la categoría que buscarían, las áreas y espacios que ofrezca el edificio, principalmente enfocado en las áreas comunes las cuales serán parte del complejo y cualquier residente puede tener acceso a ellas, así como también el factor del equipamiento que se encuentre cerca del edificio, cabe mencionar que entre más equipamiento tenga la zona donde se localice el bien inmueble, es más seguro que el precio del departamento incremente, ya que serían zonas que además de tener una buena ubicación, tiene muchos espacios que le dan valor agregado al edificio.

Al momento de tomar una decisión sobre el departamento a adquirir es importante revisar las diferentes ofertas que se encuentran en el mercado y analizar la opción que más convenga, es decir la que más se adapte a las necesidades de cada persona y tomar en cuenta todos los factores antes mencionados, así como también el tipo de financiamiento a utilizar.

Los nuevos proyectos verticales de usos mixtos ofrecen torres habitacionales, espacios para comercio, así como también áreas para renta de oficinas, por lo que adquirir un departamento hoy en día, es sin duda una gran oportunidad de inversión, siendo Monterrey una ciudad con un continuo crecimiento inmobiliario, por lo que con el paso del tiempo el valor de la propiedad se incrementará en gran medida en comparación con otros municipios.

Hoy en día la vivienda vertical sigue teniendo un crecimiento importante y en caso de seguir aumentando la oferta y demanda de vivienda en la ciudad, posiblemente se podría convertir en los próximos años en el principal tipo de vivienda en la ciudad. 


\section{REFERENCIAS}

Bautista, S. (2017). Auge de la vivienda vertical. Construcción y tecnología en concreto. 6(10). En: http://imcyc.com/revistacyt/pdf/enero_2017/ene17.pdf

Chapa M. (2018). Boom de vivienda vertical en Monterrey. Real Estate Market \& Lifestyle. Núm. 118. https://www.realestatemarket.com.mx/articulos/mercadoinmobiliario/vivienda/22598boom-de-vivienda-vertical-en-monterrey

Daúl, V. \& Plaza J. (2019). Factores que influyen en el comprador al 1 momento de adquirir un bien inmueble. Digital Publisher. Núm. 16 pp-81-93. En: https://www.593dp.com/index.php/593_Digital_Publisher/article/download/137/407/1178

García, L. (2017). Necesidades actuales del desarrollo urbano. Construcción y tecnología en concreto. 6(10). En: http://imcyc.com/revistacyt/pdf/enero 2017/ene17.pdf

Garza, A. (2018). Panorama inmobiliario en Monterrey. Real Estate Market \& Lifestyle. Núm. 118 https://www.realestatemarket.com.mx/articulos/mercado-inmobiliario/22594-panoramainmobiliario-en-monterrey

Gómez, K. (2014). Redensificación con base a la vivienda vertical: una apuesta por la calidad de vida. Revista Legado de Arquitectura y Diseño. Núm. 6. En: https://www.redalyc.org/pdf/4779/477947304006.pdf

González, J. (2017). Vivienda vertical, una apuesta muy rentable. Construcción y tecnología en concreto. 6(10). En: http://imcyc.com/revistacyt/pdf/enero 2017/ene17.pdf

Juárez, J. (2017). Uso mixto en el desarrollo inmobiliario. Construcción y tecnología en concreto. 6(10). En: http://imcyc.com/revistacyt/pdf/enero_2017/ene17.pdf

Machado, J., Miranda, R. (2004). La verticalización como resultado material de incorporación inmobiliaria en Maringa-Parana. Gestión Urbana Internacional. Núm. 57. En: https://www.redalyc.org/articulo.oa?id=19800910

Moreno, R., Alvarado, E. (2011). El entorno social y su impacto en el precio de la vivienda. Un análisis de precios hedónicos en el Área Metropolitana de Monterrey. Trayectorias, 14(33), pp. 131-147. En: http://trayectorias.uanl.mx > pdf > 7 ramsas_elias

Poeta, S., Gerhardt, T. \& Stumpf, M. (2019). Análisis de precios hedónicos de viviendas. Revista $\begin{array}{lllll}\text { Ingeniería de Construcción } & \text { RIC. }\end{array}$ https://www.ricuc.cl/index.php/ric/article/download/618/PDF\%20SPANISH

Santos, J., \& Lima, W. (2012). Análisis de los factores determinantes de la decisión de la compra del consumidor. INVENIO, pp. 55-65. En: https://www.redalyc.org/articulo.oa?id=87724146006

Valdés, A. (2017). La vivienda vertical en México: diagnóstico y tendencias. Construcción y tecnología en concreto. 6(10). En: http://imcyc.com/revistacyt/pdf/enero 2017/ene17.pdf 
Valdés, A. (2017). Ciudades verticales; ¿Utopía o realidad? Construcción y tecnología en concreto. 6(10). En: http://imcyc.com/revistacyt/pdf/enero_2017/ene17.pdf

Vázquez, R. (2018). Monterrey la ciudad más moderna del país. Real Estate Market \& Lifestyle. Núm. $\quad 118 \quad$ http://www.realestatemarket.com.mx/articulos/mercado inmobiliario/urbanismo/12429-monterrey-la-ciudad-mas-moderna-del-pais 